\title{
Effect of postoperative systemic antipsychotic therapy on psychiatric recurrence in patients with meningiomas
}

\author{
PING YANG ${ }^{1 *}$, LIANG LI $^{2,3^{*}}$, MAO-SHENG LI ${ }^{1}$, BIN ZHOU ${ }^{1}$, WEI-PING KUANG ${ }^{1}$ and HUI-YONG HUANG ${ }^{2}$ \\ ${ }^{1}$ Department of Psychiatrics, Hunan Brain Hospital, Changsha, Hunan 410007; \\ ${ }^{2}$ Provincial Key Laboratory of Traditional Chinese Medicine Diagnostics; \\ ${ }^{3}$ Department of Anatomy, Hunan University of Chinese Medicine, Changsha, Hunan 410208, P.R. China
}

Received August 19, 2017; Accepted March 28, 2018

DOI: $10.3892 / \mathrm{ol} .2018 .8753$

\begin{abstract}
A number of patients with meningiomas and psychiatric disorders will suffer psychiatric symptom recurrence following tumorectomies. The present study reported a retrospective analysis regarding 42 cases of patients with meningiomas using complete clinical follow-up data from June 2005 to June 2013. The patients were divided into the systemic antipsychotic (SP) group $(n=20)$ following 6months of postoperative SP therapy and the none-SP (NSP) group $(n=22)$, who did not receive postoperative antipsychotic treatment. Patients were assessed using the Positive and Negative Syndrome Scale (PANSS) and Brief Psychiatric Rating Scale (BPRS) at the time points of prior to surgery, immediately following surgery, and 6 months, 12 months and 3 years following surgery. The effective rate, recurrence rate, rehospitalization rate and survival analysis were calculated. The BPRS score and PANSS scores (including PANSS positive, PANSS negative, PANSS general psychopathology and PANSS overall) in the SP group at 6 months, 12 months and 3 years following surgery were significantly decreased compared with the NSP group $(\mathrm{P}<0.05)$. The effective rate was 95,90 and $90 \%$ at 6 months, 12 months and 3 years, respectively, in the SP group, whilst it was 68.2, 63.6 and 59\%, respectively, in the NSP group, which was statistically significant $\left(\chi^{2}=4.89, \chi^{2}=4.01\right.$ and $\left.\chi^{2}=5.12 ; \mathrm{P}<0.05\right)$. The recurrence rate of the SP group was 0,5 and $10 \%$ at 6 months, 12 months and
\end{abstract}

Correspondence to: Dr Hui-Yong Huang, Provincial Key Laboratory of Traditional Chinese Medicine Diagnostics, Hunan University of Chinese Medicine, 300, Xueshi Road, Changsha, Hunan 410208, P.R. China

E-mail: huanghy68@126.com

*Contributed equally

Abbreviations: SP, systemic antipsychotic; NSP, none-systemic antipsychotics; PANSS, positive and negative syndrome scale; BPRS, brief psychiatric rating scale; WHO, world health organization

Key words: meningiomas, psychiatric symptoms, antipsychotic treatment
3 years, respectively, whilst in the NSP group it was 22.7, 31.8 and $54.5 \%$, respectively, which was statistically significant $\left(\chi^{2}=5.16, \chi^{2}=4.89\right.$ and $\left.\chi^{2}=9.34 ; \mathrm{P}<0.05\right)$. The rehospitalization rate of the SP group was 0,0 and $5 \%$ at 6 months, 12 months and 3 years, respectively, whilst in the NSP group it was 13.6, 22.7 and $36.4 \%$, respectively, and the data at 12 months and 3 years was statistically significant $\left(\chi^{2}=5.16\right.$ and $\chi^{2}=6.12$; $\mathrm{P}<0.05)$. Kaplan-Meier survival analysis indicated that the accumulative survival rates of recurrence and rehospitalization in the SP group were improved compared with the NSP group. The log-rank of recurrence was $\chi^{2}=9.369(\mathrm{P}=0.002)$ and the log-rank of rehospitalization was $\chi^{2}=6.330(\mathrm{P}=0.012)$. In conclusion, postoperative SP therapy is of great importance to the consolidation of mental symptoms in patients with meningiomas and psychiatric symptoms, and it may significantly reduce the recurrence and rehospitalization rates.

\section{Introduction}

Meningiomas, originating from arachnoid cells, are a type of common intracranial tumor. They may be found at different locations in brain, especially between the brain and the skull, within ventricles, and along the spinal cord (1). These lesions can occur in people of any age but commonly present among middle-aged individuals (2). Single and multiple occurrencesmay be observed (3). The global incidence rate is preceded only by glioma, occupying $20 \%$ of intracranial primary tumor types (4). It has been demonstrated that $\sim 7.61 / 100,000$ population develop this type of tumor, accounting for $36.1 \%$ of all intracranial tumor types; the prevalence rate for meningiomas is $50.4 / 100,000$, with a notable increase in incidence rates in people aged $>65$ globally (5). The primary clinical symptoms of meningiomas are as follows: Headache; vomiting; epileptic seizure; visual diminution; walking instability; and psychiatric symptoms (6-9). Furthermore, patients with psychiatric symptoms in meningiomas are rare in clinical settings; most of them show hallucination, delusion, analepsia, agitation, impulsive behavior and insomnia (10). Some patients began with psychiatric symptoms and underwent treatment in local psychiatric hospitals; the head CT scan diagnosed the meningiomas in the end due to poor therapeutic efficacy (11). A previous study hypothesized that the psychiatric symptoms would disappear naturally once the occupying lesions were removed (12); 
however, others argued that alack of postoperative systemic antipsychotic (SP) therapy would result in recurrent psychiatric symptoms (13). In the present study, 42 cases of patients with meningiomas and psychiatric symptoms were collected, with 3 years of follow-up data. The effect of postoperative SP therapy for 6 months on psychiatric recurrence was observed.

\section{Materials and methods}

General information. The present study was a retrospective analysis approved by the ethics committee and was conducted in accordance to the relevant agreements with the Hunan Brain Hospital (Changsha, China). A total of 42 cases of patients with meningiomas in Hunan Brain Hospital from June 2005 to June 2013 were collected. The patients' written informed consent permitted the publication of the study results. All of the patients underwent computed tomography (CT) scans in the patients' local hospital. At Hunan Brain Hospital, the patients underwent $1.5 \mathrm{~T}$ enhanced magnetic resonance imaging (MRI) scans and biopsy. The CT scan, enhanced MRI and pathology data were used to confirm the diagnosis of meningioma. All the cases, having complete clinical follow-up data, were in accordance with the diagnostic criteria of International Classification of Diseases-10 for 'brain damage, dysfunction and other mental disorders caused by somatic diseases' (14). The patients were divided into two groups. The first group was the SP group $(n=20)$, undergoing 6 months of postoperative SP therapy. The other group was the none-SP (NSP) group $(n=22)$, who did not undergo postoperative antipsychotic treatment. Tumors were classified according to the World Health Organization (WHO) criteria for histological subtypes and tumor grading (grade I-III) (15).

Hematoxylin and eosin (H\&E) staining and immunohistochemistry. Tumor specimens were fixed in $4 \%$ formalin for $12 \mathrm{~h}$ at room temperature, followed by paraffin-embedded and cut into slices. The thickness of the slices used for H\&E staining and immunohistochemistry was $2.5 \mu \mathrm{m}$. H\&E staining was performed using standard histological techniques at room temperature. Sections were stained hematoxylin for $30 \mathrm{sec}$ then rinsed in $\mathrm{H}_{2} \mathrm{O}$ for $1 \mathrm{~min}$. They were stained with $1 \%$ eosin $\mathrm{Y}$ solution for $30 \mathrm{sec}$, and then dehydrated with a graded series of alcohol $(95,95,100$ and 100\%) for $30 \mathrm{sec}$ each. The alcohol was removed via washing with $95 \%$ xylene twice. A total of 1 or 2 drops of neutral gum were added and the sections were covered with a coverslip. The steps of immunohistochemistry were as follows: Sections were immersed in hydrogen peroxide $(0.3 \%$ in PBS) for $5 \mathrm{~min}$ at room temperature, followed by incubation at room temperature for $2 \mathrm{~h}$ in solution with one of the following monoclonal rabbit anti-rat antibodies (Quan Hui Co. Ltd, Guangzhou, China.): S-100 (dilution, 1:100; cat. no. 604660202); and CD34 (dilution, 1:100; cat. no. 6052344101). Then samples were incubated for $30 \mathrm{~min}$ at room temperature with the Poly-HRP Anti-Mouse/Rabbit IgG detection kit (cat. no. WP140316; Zhongshan Golden Bridge Company, Beijing, China.), according to the manufacturer's protocols, followed by detection with a DAB Kit (cat. no. K145619C; Zhongshan Golden Bridge Company, Beijing, China). Finally, sections were soaked in $95 \%$ xylene at room temperature for $2 \mathrm{~min}$ and sealed with neutral gum.
Sections were observed with a biological optical microscope (Olympus Corporation, Tokyo, Japan) at x100 magnification.

Treatment methods. All patients were treated with SP therapy prior to surgery and craniotomy to remove the tumors in Hunan Brain Hospital. Following surgical resection, 7 cases in the SP group and 9 cases in the NSP group received radiotherapy. Radiation doses were 50-60 Gy, with 2 Gy per fraction, 5 fractions per week.

In the SP group, 10 cases were treated with olanzapine 5-20 mg/day orally, 8 cases were administered quetiapine fumarate $0.2-0.6 \mathrm{~g} /$ day orally and 2 cases were treated with risperidone 3-5 mg/day orally. The SP therapy lasted for 6 months following surgical resection. In the NSP group, 13 cases were administered olanzapine $5-20 \mathrm{mg} /$ day orally, 7 cases were treated with quetiapine fumarate 0.2-0.6 g/day orally and 2 cases were treated with risperidone $3-5 \mathrm{mg}$ /day orally; however, all of them reduced the dose or withdrew the antipsychotic drugs voluntarily. Treatments varied according to the prescriptions of physicians.

Follow-up and effective rate evaluation. All patients had complete follow-up records. They were followed up at 6 months, 12 months and 3 years following surgical resection. Psychiatric symptoms were evaluated via the Positive and Negative Syndrome Scale (PANSS) (16) and Brief Psychiatric Rating Scale (BPRS) (17) at 5 different time points (prior to surgery, immediately following surgery, and 6 months, 12 months and 3 years following surgery). The evaluation was assessed by two experienced physicians from Hunan Brain Hospital who were blind to the treatment protocols. The effective rate was assessed with BPRS. A reduction rate $\geq 75 \%$ represented recovery, $50-74 \%$ represented significant progress, $25-49 \%$ represented progress and $<25 \%$ represented no progress. The clinical criteria of recovery were as follows: Symptoms disappeared completely; insight recovered; and social adaptability improved notably, and that all of these lasted for $\geq 6$ months.

Statistical analysis. Statistical analysis was performed with SPSS (version 23.0; IBM Corp., Armonk, NY, USA). The quantitative data are presented as mean \pm standard deviation (SD), and the Student's t-test was used for intergroup comparisons. The $\chi^{2}$ test or Fisher's exact test were used for intergroup comparisons of enumeration data, including: The recurrence rate at 6 months; and the rehospitalization rate at 12 months. Kaplan-Meier survival analysis with a log-rank test was used for recurrence and rehospitalization. $\mathrm{P}<0.05$ was considered to indicate a statistically significant difference.

\section{Results}

General disease presentation. The SP group included 8 males and 12 females aged 16-52 years, and the mean \pm SD patient age was $39.50 \pm 8.72$ years. The duration of disease ranged from 1-40 months, and the mean \pm SD duration was $18.35 \pm 12.87$ months. The mean \pm SD radiation doses were $54.13 \pm 3.76 \mathrm{~Gy}$. The patients presented with different primary symptoms, including: 7 cases of hallucination; 8 cases of delusion; and 5 cases of impulsive and aggressive behavior. The 

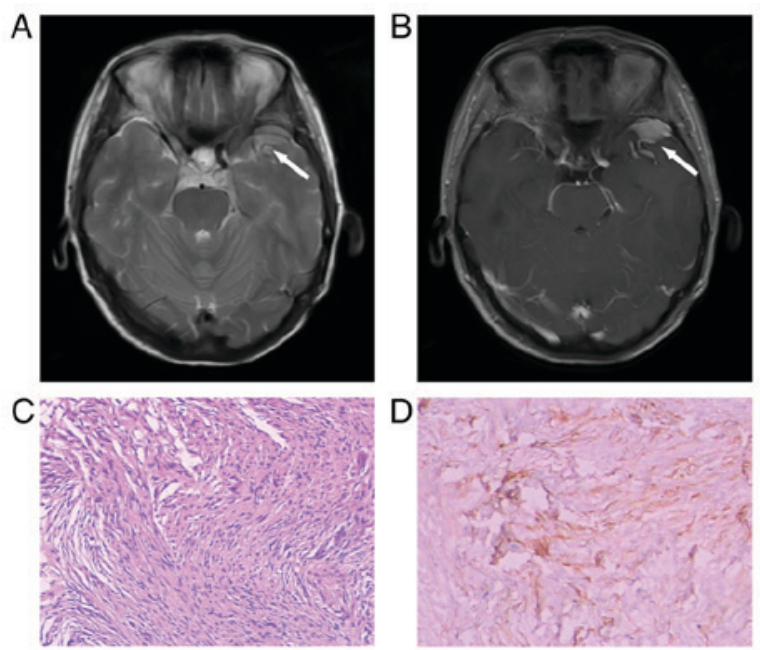

Figure 1. Local staining of a 46-year-old female patient (systemic antipsychotic group) diagnosed with fibrous meningioma in the left temporal lobe, and primary symptoms of hallucination. The diameter of tumor was $\sim 2.3 \mathrm{~cm}$. (A) The white arrow represents the space-occupying lesion on T2-weighted images prior to surgery (axial view). (B) The white arrow represents the space-occupying lesion on enhanced images using gadopentetate dimeglumine as a contrast agent prior to surgery (axial view). (C) The pathology slice indicated fibrous type (hematoxylin and eosin staining; magnification, $\mathrm{x} 100$ ). (D) The pathology slice indicated the positive expression of S-100 (immunohistochemistry; magnification, x100).

NSP group included 9 males and 13 females; aged 19-55 years, and the mean \pm SD patient age was $39.14 \pm 8.71$ years. The duration of disease ranged from 0.5-36 months, and the mean $\pm \mathrm{SD}$ duration was $21.98 \pm 11.00$ months. The mean \pm SD radiation doses were $54.56 \pm 2.96 \mathrm{~Gy}$. They manifested different primary symptoms, including: 8 cases of hallucination; 8 cases of delusion; and 6 cases of impulsive and aggressive behavior. The aforementioned data had no statistical significance $(\mathrm{P}>0.05)$. The radiation rate between the SP and NSP groups had no statistical significance $(\mathrm{P}>0.05)$.

Lesions were located in different areas in the SP group, including: 8 cases in the frontal lobe; 9 cases in the temporal lobe; 1 case in the temporo-occipital area; 1 case near the sagittal sinus in the parietal lobe; and 1 case in the pontocerebellar peduncle. The mean \pm SD diameter of the tumors was $3.85 \pm 0.55 \mathrm{~cm}$. The NSP group included: 9 cases located in the frontal lobe; 9 cases in the temporal lobe; 1 case in the parieto-occipital area; 2 cases in the sphenoidal crest; and 1 case in the posterior cranial fossa. The mean \pm SD diameter of the tumors was $4.13 \pm 0.54 \mathrm{~cm}$. The postoperative pathology results demonstrated that the SP group included 17 cases of WHO grade I (5 meningothelial type, 4 mixed type, 5 fibrous type and 3 psammomatous type) and 3 cases of WHO grade II (all atypical type). The NSP group was composed of 18 cases of WHO grade I (4 meningothelial type, 5 mixed type, 5 fibrous type and 4 psammomatous type) and 4 cases of WHO grade II (atypical type). The aforementioned data had no statistical significance $(\mathrm{P}>0.05$; Figs. 1 and 2$)$.

Comparison of PANSS and BPRS. There was no significant difference in PANSS and BPRS between the two groups prior to and immediately following surgical resection. The BPRS and PANSS scores (including PANSS positive, PANSS
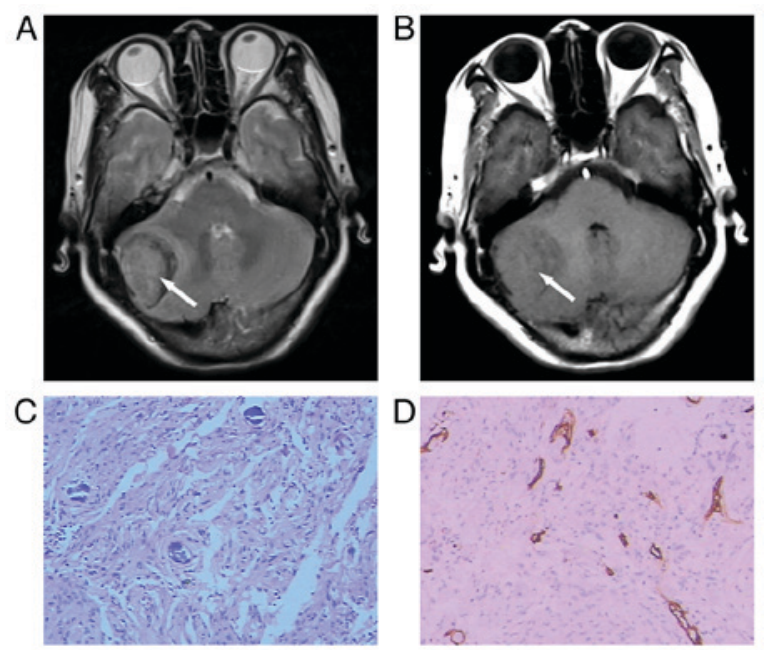

Figure 2. Local staining of a 62-year-old female patient (none-systemic antipsychotics group) diagnosed with mixed meningioma in the right posterior cranial fossa, and symptoms of anxiety, depression and insomnia. The largest diameter of tumor was $\sim 2.1 \mathrm{~cm}$. The symptoms improved notably following surgery, and the patient withdrew from treatment one month following leaving the hospital. The psychiatric symptoms recurred 5 months following surgery. (A) The white arrow represents the space-occupying lesion on T2-weighted images prior to surgery (axial view). (B) The white arrow represents the space-occupying lesion on enhanced images using gadopentetate dimeglumine as a contrast agent prior to surgery (axial view). (C) The pathology slice indicated mixed type (hematoxylin and eosin staining; magnification, x100). (D) The pathology slice indicated the positive expression of CD34 in the vessel wall (immunohistochemistry; magnification, x100).

negative, PANSS general psychopathology and PANSS overall) in the SP group at 6 months, 12 months and 3 years were significantly decreased compared with the NSP group $(\mathrm{P}<0.05$; Figs. 3 and 4$)$.

Follow-up results. All patients were followed up at 6 months, 12 months and 3 years. The effective rate was 95, 90 and $90 \%$ at 6 months, 12 months and 3 years, respectively, in the SP group, whilst it was $68.2,63.6$ and $59 \%$, respectively, in the NSP group, which was significantly different $\left(\chi^{2}=4.89, \chi^{2}=4.01\right.$ and $\left.\chi^{2}=5.12 ; \mathrm{P}<0.05\right)$. The recurrence rate in the SP group was 0,5 and $10 \%$ at 6 months, 12 months and 3 years, respectively, whilst in the NSP group it was 22.7, 31.8 and 54.5\%, respectively, which was statistically significant $\left(\chi^{2}=5.16, \chi^{2}=4.89\right.$ and $\left.\chi^{2}=9.34 ; \mathrm{P}<0.05\right)$. The rehospitalization rate of the SP group was 0,0 and $5 \%$ at 6 months, 12 months and 3 years, respectively, whilst in the NSP group it was 13.6, 22.7 and $36.4 \%$, respectively, and the data at 12 months and 3 years was statistically significant $\left(\chi^{2}=5.16\right.$ and $\chi^{2}=6.12 ; \mathrm{P}<0.05$; Table I).

Kaplan-Meier survival analysis in recurrence and rehospitalization. The accumulative survival rates of recurrence and rehospitalization in the SP group were significantly improved compared with the NSP group. The log-rank of recurrence was $\chi^{2}=9.369(\mathrm{P}=0.002)$ and the log-rank of rehospitalization was $\chi^{2}=6.330(\mathrm{P}=0.012$; Fig. 5).

\section{Discussion}

The majority of meningiomas are benign tumors (18). They grow slowly and the disease course usually lasts for 

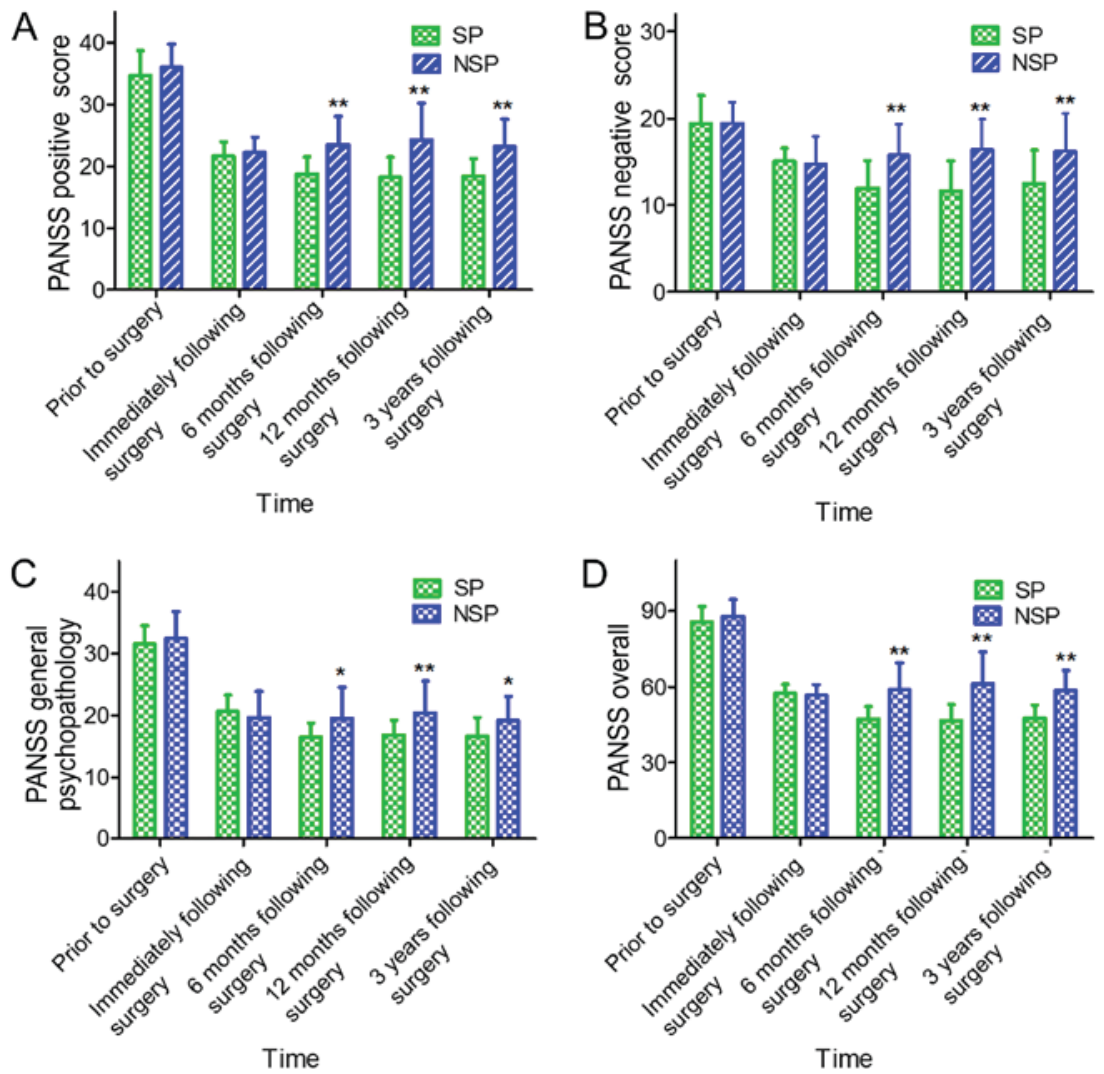

Figure 3. PANSS scores, including (A) PANSS positive, (B) PANSS negative, (C) PANSS general psychopathology and (D) PANSS overall at the time points of prior to surgery, immediately following surgery, and 6 months, 12 months and 3 years following surgery. Data are expressed as mean \pm standard deviation. ${ }^{*} \mathrm{P}<0.05$ and ${ }^{* *} \mathrm{P}<0.01$ vs. SP group. SP, systemic antipsychotic; NSP, none-SP; PANSS, positive and negative syndrome scale.

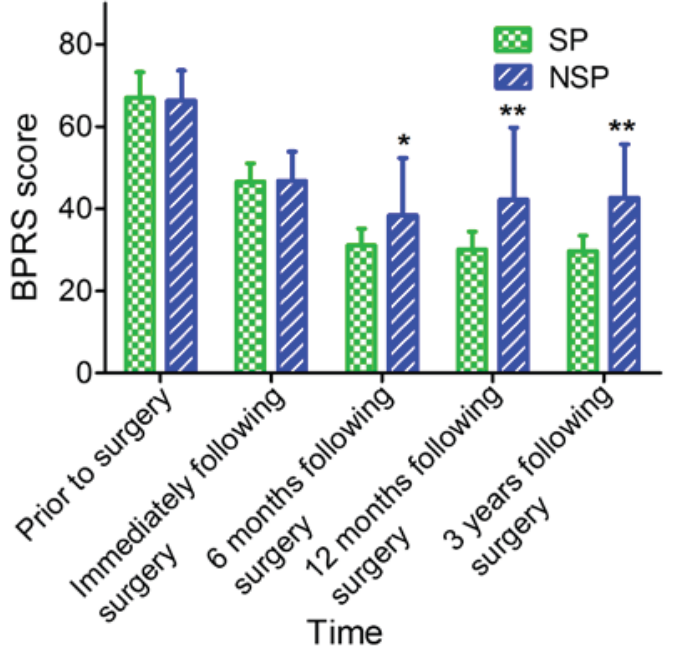

Figure 4. BPRS score at the time points of prior to surgery, immediately following surgery, and 6 months, 12 months and 3 years following surgery. Data are expressed as mean \pm standard deviation. ${ }^{*} \mathrm{P}<0.05$ and ${ }^{* *} \mathrm{P}<0.01$ vs. SP group. SP, systemic antipsychotic; NSP, none-SP; BPRS, brief psychiatric rating scale.

2-4 years $(19,20)$. Detection is difficult at the early stages due to patients being asymptomatic (21). It is only when the activity of the nervous system becomes disrupted that the serious clinical symptoms appear, thereby alerting clinicians (22). The primary symptoms include: Chronic headaches; hallucination; delusion; epilepsy; visual diminution or loss; and impaired coordination (23-25). Different locations of the meningiomas indicate various clinical manifestations (26), and it has been demonstrated that the incidence of patients with meningiomas and psychiatric symptoms is rare $(27,28)$. In the present study, 30 of all 42 patients had a history of treatment in other hospitals. Among them, 9 patients were diagnosed with mental disorders at local hospitals for initial psychiatric symptoms, and were treated with antipsychotics only. The diagnosis of the meningiomas and treatment were delayed until serious clinical symptoms emerged, which then resulted in additional examination and eventual disease confirmation.

In the present study, there were 35 patients, in which the meningiomas located in the frontal and temporal lobes were present in $83.3 \%$ of all 42 patients. It has been determined that the primary symptoms of frontotemporal tumors include: Hallucination; delusion; impulsive aggressive behaviors; and cognitive handicap (29-31), and it was considered that these mental symptoms were closely associated with the location of the occupying lesions (32). The treatment of patients with meningiomas and psychiatric symptoms in neurosurgery is difficult, for example, there were 10 patients in the present study who could not undergo the surgical resection because of the repeated symptoms of agitation and aggressive behavior; therefore, doctors had to control their symptoms with the administration of psychiatric treatment followed by the operation. Accompanied by the improved mental symptoms, a debate exists between neurosurgeons and psychiatrists concerning the application of antipsychotic drugs in patients with meningiomas. The majority of the neurosurgeons believe 
Table I. Comparison of follow-up results.

\begin{tabular}{lccccc}
\hline Group & Time following surgery & $\mathrm{n}$ & Effective rate $(\%)$ & Recurrence rate $(\%)$ & Rehospitalization rate (\%) \\
\hline SP & 6 months & 20 & 95.0 & 0.0 & 0.0 \\
& 12 months & 20 & 90.0 & 5.0 & 0.0 \\
& 3 years & 20 & 90.0 & 10.0 & 5.0 \\
NSP & 6 months & 22 & $68.2^{\mathrm{a}}$ & $22.7^{\mathrm{a}}$ & 13.6 \\
& 12 months & 22 & $63.6^{\mathrm{a}}$ & $31.8^{\mathrm{a}}$ & $22.7^{\mathrm{a}}$ \\
& 3 years & 22 & $59.0^{\mathrm{a}}$ & $54.5^{\mathrm{b}}$ & $36.4^{\mathrm{b}}$ \\
\hline
\end{tabular}

$\chi^{2}$ test has been use. ${ }^{a} \mathrm{P}<0.05$ and ${ }^{\mathrm{b}} \mathrm{P}<0.01$ vs. SP group. SP, systemic antipsychotic; NSP, none-SP.
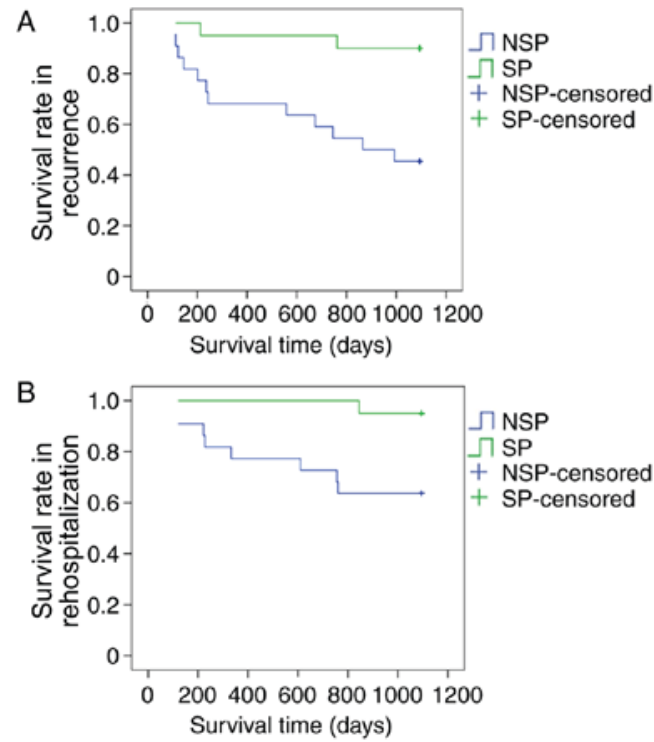

Figure 5. (A) Kaplan-Meier survival rate in recurrence displays the difference between the SP and NSP groups. (B) Kaplan-Meier survival rate in rehospitalization displays the difference between the SP and NSP groups. SP, systemic antipsychotic; NSP, none-SP.

patients can withdraw the antipsychotic drugs immediately following surgery. While in clinic, this could result in severe mental symptoms and/or recurrent attacks which reduce the quality of life (13).

At present, there are no studies focusing on the duration of postoperative SP therapy for patients with meningiomas and psychiatric symptoms. A number of previous studies have suggested that antipsychotic drugs maybe gradually reduced until withdrawal, following the slow improvement of psychiatric symptoms $(33,34)$. However, in a clinical setting, it was determined that the withdrawal of antipsychotic drugs following surgical resection may result in the recurrence of psychiatric symptoms; subsequently, the patients would have to receive the antipsychotic treatment again, which may be associated with local metabolic imbalances caused by the pace-occupying lesions (35).

Therefore, the present study retrospectively analyzed the patients with meningiomas and psychiatric symptoms treated at Hunan Brain Hospital. All the patients were divided into SP and NSP groups according to their treatment, and follow-up was conducted for 3 years. The psychiatric symptoms were compared using the PANSS, BPRS, effective rate, recurrence rate and rehospitalization rate between these two groups.

All of the patients received tumorectomy, and 16 of 42 patients were treated with radiotherapy. The expression of S-100 or CD34 in the tumors was detected with immunohistochemistry. In the present study, as the focus was not on the diagnosis of meningioma but on the postoperative SP therapy, the immunohistochemistry results were used to confirm the diagnosis of meningioma and not for statistical analysis. Furthermore, the patients failed to be analyzed for relevant biochemical indices, including NSE, S-100 and CEA, due to funding limitations. This was a limitation of the present study. Despite this, the results of the CT and enhanced MRI scans and pathology data confirmed the diagnosis of meningioma. It was determined that the BPRS score and PANSS scores (including PANSS positive, PANSS negative, PANSS general psychopathology and PANSS overall) in the SP group at 6 months, 12 months and 3 years were significantly decreased compared with the NSP group, all of which were statistically significant. The follow-up results demonstrated that the effective rate was significantly increased, whilst the recurrence and rehospitalization rates were significantly decreased in the SP group, compared with the NSP group at 6 months, 12 months and 3 years. The Kaplan-Meier survival analysis demonstrated that the accumulative survival rates of recurrence and rehospitalization in the SP group were improved compared with the NSP group. Postoperative SP therapy is of great importance to the consolidation of mental symptoms in patients with meningiomas and psychiatric symptoms, and it may significantly reduce the recurrence and rehospitalization rates.

The results of the present study demonstrated that postoperative SP therapy for 6 months is necessary for the prevention of recurrence and reducing the rehospitalization rate in patients with meningiomas and psychiatric symptoms. Additional studies with Magnetic Resonance Spectrum Imaging of space-occupying lesions are required, which would subsequently clarify whether the psychiatric symptoms are associated with nervous metabolic abnormalities following surgical resection.

\section{Acknowledgements}

Not applicable. 


\section{Funding}

This work was supported by grants from the National Natural Science Foundation of China (grant nos. 81603512 and 81373551) and the Science \& Research Project of Hunan Provincial Education Department (grant no. 15C1051).

\section{Availability of data and materials}

The datasets used and/or analyzed during the current study are available from the corresponding author on reasonable request.

\section{Authors' contributions}

LL and HYH designed the study. PY and MSL performed the experiments. BZ participated in study design and data collection. WPK was in charge of statistical analysis. PY wrote the manuscript and LL revised the manuscript.

\section{Ethics approval and consent to participate}

This present study was approved by the ethics committee and was conducted in accordance with the relevant agreements of the Hunan Brain Hospital (Changsha, China, No 2012-8). Written informed consent was obtained from all participants prior to their inclusion within the study.

\section{Consent for publication}

The patients' written informed consent permitted the publication of the study results. Identifying information about the patients has been removed.

\section{Competing interests}

The authors declare that they have no competing interests.

\section{References}

1. Shintaku M, Hashimoto $\mathrm{K}$ and Okamoto S: Intraventricular meningioma with anaplastic transformation and metastasis via the cerebrospinal fluid. Neuropathology 27: 448-452, 2007.

2. Saha R, Jakhar K and Kumar R: Sphenoid wing meningioma presenting as cognitive impairment. Shanghai Arch Psychiatry 28: 173-176, 2016.

3. Terrier LM and François P: Multiple meningiomas. Neurochirurgie 62: 128-135, 2016 (In French).

4. Hwang WL, Marciscano AE, Niemierko A, Kim DW, Stemmer-Rachamimov AO, Curry WT, Barker FG II, Martuza RL, Loeffler JS, Oh KS, et al: Imaging and extent of surgical resection predict risk of meningioma recurrence better than WHO histopathological grade. Neuro Oncol 18: 863-872, 2016.

5. Silva CB, Ongaratti BR, Trott G, Haag T, Ferreira NP, Leaes CG, Pereira-Lima JF and Oliveira Mda C: Expression of somatostatin receptors (SSTR1-SSTR5) in meningiomas and its clinicopathological significance. Int J Clin Exp Pathol 8: 13185-13192, 2015.

6. Aizer AA, Bi WL, Kandola MS, Lee EQ, Nayak L, Rinne ML, Norden AD, Beroukhim R, Reardon DA, Wen PY, et al: Extent of resection and overall survival for patients with atypical and malignant meningioma. Cancer 121: 4376-4381, 2015.

7. Deng WS, Zhou XY, Li ZJ, Xie HW, Fan MC and Sun P: Microsurgical treatment for central gyrus region meningioma with epilepsy as primary symptom. J Craniofac Surg 25: $1773-1775,2014$
8. Wang DJ, Xie Q, Gong Y, Wang Y, Cheng HX, Mao Y, Zhong P, Huang FP, Zheng K, Wang YF, et al: Secretory meningiomas: Clinical, radiological and pathological findings in 70 consecutive cases at one institution. Int J Clin Exp Pathol 6: 358-374, 2013.

9. Xu P, Luo H, Huang GL, Yin XH, Luo SY and Song JK: Exposure to ionizing radiation during dental $\mathrm{X}$-rays is not associated with risk of developing meningioma: A meta-analysis based on seven case-control studies. PLoS One 10: e0113210, 2015.

10. Tringale KR, Wilson BR, Hirshman B, Zhou T, Folsom D, Norman MA, Grant I, Chen CC and Carter BS: Psychiatric disease preceding intracranial tumor diagnosis: Investigating the association. Prim Care Companion CNS Disord 18: 2016.

11. Wang RT: Meningiomas begin with psychiatric symptoms: A case report. Chin Med Herald 7: 104, 2010 (In Chinese).

12. Yakhmi S, Sidhu BS, Kaur J and Kaur A: Diagnosis of frontal meningioma presenting with psychiatric symptoms. Indian J Psychiatry 57: 91-93, 2015.

13. Zhou B, Kuang WP, Huang HX, Li B, Zhu Y, Chen XF, Yan L and Yang P: Clinical research to the Meningioma patients with psychiatric symptoms in 16 cases. Chin J Clin Neurosci 24: 553-556, 2016 (In Chinese).

14. World Health Organization: The ICD-10 classification of mental and behavioural disorders: Clinical descriptions and diagnostic guidelines. Geneva, 1992.

15. Louis DN, Ohgaki H, Wiestler OD and Cavenee WK: WHO classification of tumours of the central nervous system. IARC Press, Lyon, 2007.

16. Horga G, Cassidy CM, Xu X, Moore H, Slifstein M, Van Snellenberg JX and Abi-Dargham A: Dopamine-related disruption of functional topography of striatal connections in unmedicated patients with schizophrenia. JAMA Psychiatry 73: 862-870, 2016

17. Lee SH, Niznikiewicz M, Asami T, Otsuka T, Salisbury DF, Shenton ME and McCarley RW: Initial and progressive gray matter abnormalities in insular gyrus and temporal pole in First-episode schizophrenia contrasted with First-episode affective psychosis. Schizophr Bull 42: 790-801, 2016.

18. Murnyák B, Csonka T and Hortobágyi T: Molecular pathology of meningiomas. Ideggyogy Sz 68: 292-300, 2015 (In Hungarian).

19. Du Z, Abedalthagafi M, Aizer AA, McHenry AR, Sun HH, Bray MA, Viramontes O, Machaidze R, Brastianos PK, Reardon DA, et al: Increased expression of the immune modulatory molecule PD-L1 (CD274) in anaplastic meningioma. Oncotarget 6: 4704-4716, 2015.

20. Tang H, Gong Y, Mao Y, Xie Q, Zheng M, Wang D, Zhu H, Wang X, Chen H, Chen X and Zhou L: CD133-positive cells might be responsible for efficient proliferation of human meningioma cells. Int J Mol Sci 13: 6424-6439, 2012.

21. Jurinovic P, Bulicic AR, Marcic M, Mise NI, Titlic M and Suljic E: Foramen magnum meningioma: A case report and review of literature. Acta Inform Med 24: 74-77, 2016.

22. Guo XB and Wang ZM: Mental disorder due to meningiomas: A case report. J Clin Psychiatry 17: 273-273, 2007 (In Chinese).

23. Serna E, Morales JM, Mata M, Gonzalez-Darder J, San Miguel T, Gil-Benso R, Lopez-Gines C, Cerda-Nicolas M and Monleon D: Gene expression profiles of metabolic aggressiveness and tumor recurrence in benign meningioma. PLoS One 8: e67291, 2013.

24. Oyama H, Kito A, Maki H, Hattori K, Noda T and Wada K: Postoperative recovery from unilateral blindness caused by tuberculum sellae meningioma. Nagoya J Med Sci 74: 181-187, 2012.

25. Jung HW, Lee HC, Kim JH, Jang HM, Moon JH, Sur JH, Ha J and Jung DI: Imatinib mesylate plus hydroxyurea chemotherapy for cerebellar meningioma in a Belgian Malinois dog. J Vet Med Sci 76: 1545-1548, 2014.

26. Wiemels J, Wrensch M and Claus EB: Epidemiology and etiology of meningioma. J Neurooncol 99: 307-314, 2010.

27. Madhusoodanan S, Ting MB, Farah T and Ugur U: Psychiatric aspects of brain tumors: A review. World J Psychiatry 5: 273-285, 2015.

28. Madhusoodanan S, Patel S, Reinharth J, Hines A and Serper M: Meningioma and psychiatric symptoms: A case report and brief review. Ann Clin Psychiatry 27: 126-133, 2015.

29. Cipolotti L, Healy C, Chan E, Bolsover F, Lecce F, White M, Spano B, Shallice T and Bozzali M: The impact of different aetiologies on the cognitive performance of frontal patients. Neuropsychologia 68: 21-30, 2015. 
30. James AI, Bohnke JR, Young AW and Lewis GJ: Modelling verbal aggression, physical aggression and inappropriate sexual behaviour after acquired brain injury. Proc Biol Sci: 282 20150711, 2015.

31. Yu G, Bao WM, Mao Y, Guo QH, Xie R, Shen C, Gao C and Ji YD: Study of executive functions of patients with tumor in both right and left frontal lobe. Chin J Clin Neurosci 18: 54-57, 2010 (In Chinese).

32. Lehmann G, Bremond J, Rabaud C and Paillas JE: Space-occupying lesions of the occupital lobe of the cerebral cortex. Neurochirurgie 21: 55-79, 1975 (In French).

33. Wei YH and Pan RD: Clinical analysis to viral encephalitis with initial psychiatric symptoms in 95 cases. Chin J Nerv Ment Dis 33: 45-47, 2007 (In Chinese).
34. Więdłocha M, Marcinowicz P and Stanczykiewicz B: Psychiatric aspects of herpes simplex encephalitis, tick-borne encephalitis and herpes zoster encephalitis among immunocompetent patients. Adv Clin Exp Med 24: 361-371, 2015.

35. Zhao HH, You NX, Yan YX, Di LB and Guan YR: Effect of olanzapine to mental disorders caused by organic and somatic diseases. Chin J Psychiatry 34: 225, 2001 (In Chinese).

c) (i) $\ominus$ This work is licensed under a Creative Commons cc) Attribution-NonCommercial-NoDerivatives 4.0 International (CC BY-NC-ND 4.0) License. 